\title{
The Assessment and Management of the Arthritic Knee: An Update
}

Shehzaad Khan ${ }^{1}$, Peter C. Logan ${ }^{2}$, Ajay Asokan ${ }^{3}$, Charles Handford ${ }^{4}$, Thomas Moores ${ }^{2}$

1. Trauma and Orthopaedics, Basildon and Thurrock University Hospital, London, GBR 2. Trauma and Orthopaedics, Walsall Manor Hospital, Birmingham, GBR 3. Trauma and Orthopaedics, University College London Hospital, London, GBR 4. Trauma and Orthopaedics, Queen Elizabeth Hospital, Birmingham, GBR

Corresponding author: Peter C. Logan, peter.logan3@nhs.net

\begin{abstract}
Musculoskeletal disorders represent a significant primary care burden. Presentations pertaining to the painful knee are associated with a wide array of differentials; however, among these, osteoarthritis (OA) is the most common one in patients older than 45 years. We have found that a significant number of onward secondary care referrals are misdirected. Therefore, there is a need for a comprehensive assessment and workup to ensure holistic patient care and timely input from specialist services. In this article, we highlight an approach to the management of the arthritic knee.
\end{abstract}

Categories: Family/General Practice, Orthopedics

Keywords: knee osteoarthritis/ koa, knee arthroplasty, conservative management

\section{Introduction And Background}

Musculoskeletal disorders constitute a third of general practice (GP)/family medicine consultations. A significant number of GP referrals for specialist orthopaedic review are deemed to be inappropriate or misdirected, with only $58 \%$ of those received considered necessary [1]. The most common site for musculoskeletal pathology presenting in primary care is the spine, followed by the knee [1]. Osteoarthritis $(\mathrm{OA})$ is the most common cause of knee pain in patients over the age of 45 years.

In this article, we outline an assessment approach for patients with suspected OA of the knee, discuss the indications for referrals to specialist services, and present up-to-date evidence regarding the topic.

\section{Review}

\section{Diagnosis}

The differentials for adults presenting with knee pain are wide and extensive (Table 1). However, among these, $\mathrm{OA}$ is the most common one in patients over the age of 45 years, with a clear female preponderance

Review began 09/27/2020 Review ended 11/03/2020 Published 11/19/2020

\section{() Copyright 2020}

Khan et al. This is an open access article distributed under the terms of the Creative Commons Attribution License CC-BY 4.0., which permits unrestricted use, distribution, and reproduction in any medium, provided the original author and source are credited.
[2]. The National Institute for Health and Care Excellence (NICE) has published guidelines on the assessment and management of OA, stating that OA can be diagnosed clinically if a person is over the age of 45 years, has activity-related joint pain, and has either a) no morning joint stiffness, or b) morning stiffness lasting no longer than 30 minutes [2]. 


\section{Cureus}

\section{Traumatic}

Sprain

Patella tendonitis

Bone bruising

Ligament rupture

Meniscal tear

Osteochondral defect

Patella dislocation

\section{Atraumatic}

\section{Osteoarthritis}

\section{Gout/pseudogout}

Inflammatory arthritis

Bursitis

Septic arthritis

Avascular necrosis

Osteochondritis dissecans

Fracture

TABLE 1: A list of differential diagnoses to consider for a painful knee in an adult

Radiographs are the gold standard for the first-line investigation of knee pain. The following views should be included: weight-bearing anterior-posterior (AP), lateral, and merchant views. When combined with a physical examination, the sensitivity and specificity of diagnosing OA in the knee are $91 \%$ and $86 \%$, respectively [3].

MRI should not be requested routinely, except in cases of diagnostic uncertainty, or when there is a high clinical suspicion of a possible alternative diagnosis, e.g., osteochondritis dissecans or avascular necrosis. MRI is particularly unhelpful in the elderly population with suspected OA, where incidental pathological meniscal findings are very common but not clinically significant $[4,5]$.

\section{Management}

NICE proposes a holistic approach to the management of OA (Figure 1). 


\section{Cureus}

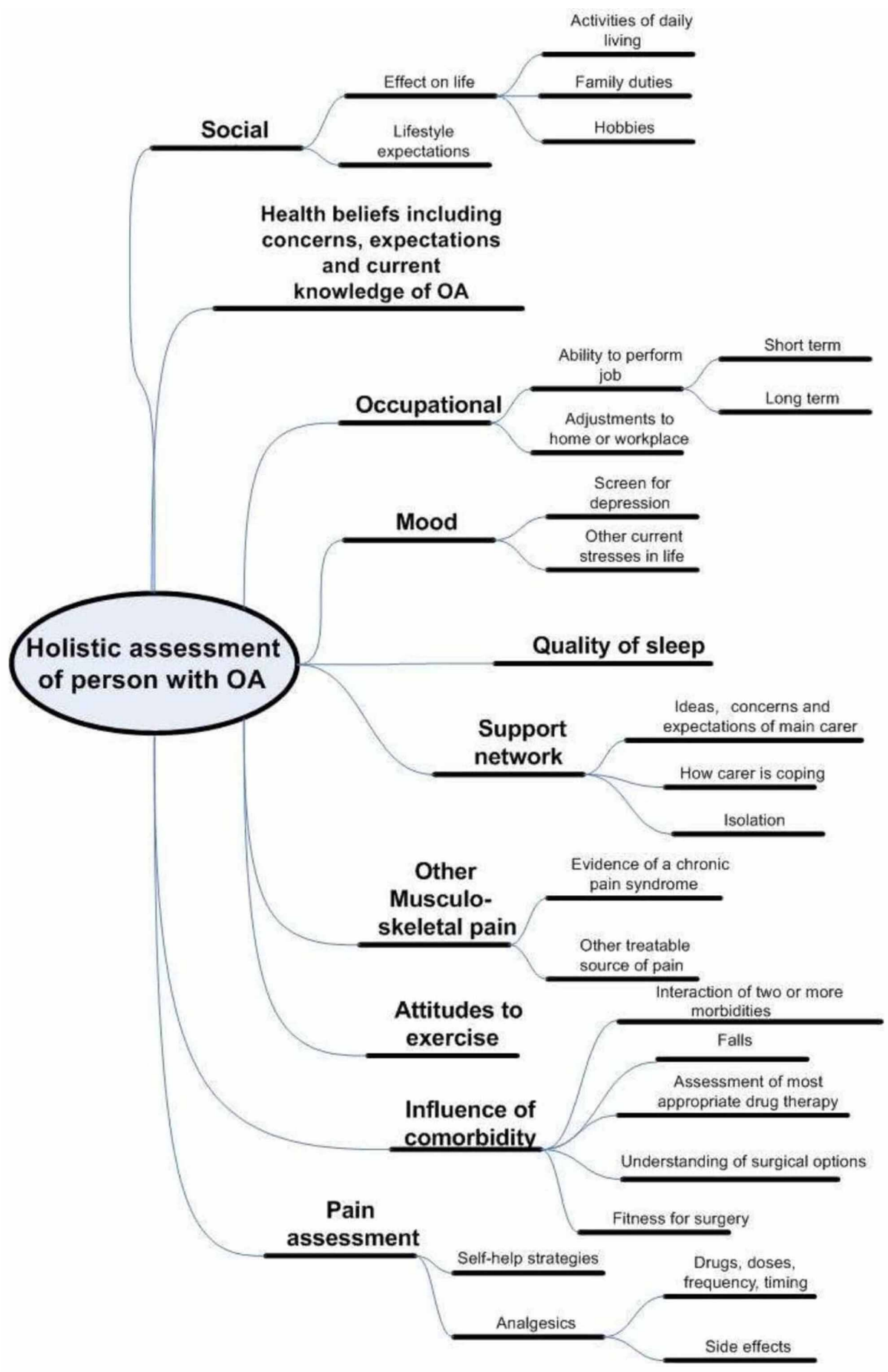

FIGURE 1: NICE guidelines on the holistic assessment of a patient with OA

NICE: the National Institute for Health and Care Excellence; OA: osteoarthritis

Non-surgical options that should be initiated in the primary care setting

Weight loss: as trivial as it sounds, this should be one of the first-line treatments offered for symptomatic $\mathrm{OA}$. A reduction in weight decreases the reaction forces across the joint, thereby decreasing pain. Weight loss should be offered before starting weight-bearing exercise to preserve joint integrity. Body mass index (BMI) of the patients must be clearly documented in any onward referrals, as some local organisations (Care Commissioning Groups) will have a BMI cut-off for certain procedures; e.g., no joint replacements with a BMI of $>35 \mathrm{~kg} / \mathrm{m}^{2}$. This is due to the increased risk of infection associated with excess adiposity. 
Physical rehabilitation and exercise: in addition to the benefits accorded by weight loss, exercise and thighstrengthening have also been shown to confer further cumulative benefits. There is level 1 evidence to support improved pain and function after 12 dedicated sessions. The Osteoarthritis Research Society International (OARSI) has endorsed the use of land-based exercise programs as a core treatment for knee OA [6]. Aquatic options should be suggested for patients with serious mobility and functional limitations. This has been quoted as a 'level 1B' therapy in knee OA, with $>75 \%$ in favour of its use based on a systematic review and analysis [6].

Knee braces and walking aids: offloading knee braces have been shown to be especially useful in unicompartmental OA. However, insoles and wedges have had no significant effects on symptoms.

Pharmacological options: non-steroidal anti-inflammatory drugs (NSAIDs), highly selective cyclooxygenase2 (COX-2) inhibitors, and tramadol have shown the best evidence for better efficacy and symptom control, as advised by the American Academy of Orthopaedic Surgeons (AAOS). This view has been further supported by the OARSI guidelines, which has found the use of topical NSAID therapy to be a level 1A therapy [6]. These can be advocated in the absence of contraindications, e.g., use of NSAIDs in peptic ulcer disease, and in such circumstances, the concurrent use of a gastro-protective proton pump inhibitor (PPI) can be added.

Alternative treatment methods: options such as glucosamine, chondroitin, viscoelastic joint injections, and acupuncture are controversial and AAOS has provided strong evidence advocating against their use.

Intra-articular corticosteroids: corticosteroid therapy has been shown to provide good short-term relief by directly reducing joint inflammation, without significant systemic side effects. While the risks include tendon rupture, articular cartilage degeneration, and septic arthritis, the use of steroid injections has become increasingly popular as more GPs are trained in its use. Up to $96 \%$ of patients have reported satisfaction with corticosteroid injections and a resultant reduction in pain scores. Two key points to note are the concentration of the local anesthetic being used and the timing of the injection:

A) a high dose of local anesthetic is associated with increased chondrolysis and we, therefore, recommend using a diluted dose, e.g., $0.25 \%$ levobupivacaine.

B) higher rates of post-total knee arthroplasty infections have been noted in patients who had received an intra-articular corticosteroid injection within three to six months of their operation [7]. Roughly 6-9\% of patients receive an intra-articular injection prior to the referral [2]. Therefore, any patient who seeks an orthopaedic opinion regarding surgical interventions should refrain from receiving an intra-articular injection.

Surgical Options

Joint preservation: mechanical symptoms such as 'locking' or 'catching' have traditionally qualified for arthroscopic procedures, e.g., arthroscopic meniscal debridement. However, evidence has shown that performing such procedures in OA knees results in little to no improvement in symptoms and function when compared to placebo or conservative options [8]. We recommend that patients in this category be referred for physiotherapy instead.

Joint arthroplasty: currently, indications that are considered to refer a patient for arthroplasty are as follows: severe and debilitating pain, especially at night; exhaustion of all conservative measures; functional impairment to the quality of life; radiographic evidence of OA. BMI also plays a role in local organisations' decision-making, with many declining to operate on patients with a BMI greater than $40 \mathrm{~kg} / \mathrm{m}^{2}$ due to their complication profile, except in individually requested circumstances [9]. Oxford Knee Scores should be measured for all patients to benchmark their symptoms pre- and post-intervention. However, they should not be used to determine a patient's suitability for surgery [10].

\section{Conclusions}

Based on our findings, the key take-aways are as follows: OA of the knee is a common diagnosis made in primary care. Many of these patients can be managed very comfortably in the community. However, using the above-mentioned criteria and in line with up-to-date evidence, we can highlight the cases of more urgent patients who may require surgical intervention and thereby reduce the referral rates. This would result in shorter waiting lists for outpatient appointments and a better patient journey and outcomes.

\section{Additional Information}

\section{Disclosures}

Conflicts of interest: In compliance with the ICMJE uniform disclosure form, all authors declare the following: Payment/services info: All authors have declared that no financial support was received from any organization for the submitted work. Financial relationships: All authors have declared that they have 
no financial relationships at present or within the previous three years with any organizations that might have an interest in the submitted work. Other relationships: All authors have declared that there are no other relationships or activities that could appear to have influenced the submitted work.

\section{References}

1. Speed CA, Crisp AJ: Referrals to hospital-based rheumatology and orthopaedic services: seeking direction Rheumatology (Oxford). 2005, 44:469-71. 10.1093/rheumatology/keh504

2. National Institute for Health and Clinical Excellence: osteoarthritis: care and management . (2014). Accessed: February 11, 2020: https://www.nice.org.uk/guidance/cg177/chapter/1-Recommendations.

3. Conaghan PG, Dickson J, Grant RL; Guideline Development Group: Care and management of osteoarthritis in adults: summary of NICE guidance. BMJ. 2008, 336:502-3. 10.1136/bmj.39490.608009.AD

4. Englund M, Guermazi A, Gale D, Hunter DJ, Aliabadi P, Clancy M, Felson DT: Incidental meniscal findings on knee MRI in middle-aged and elderly persons. N Engl J Med. 2008, 359:1108-15. 10.1056/NEJMoa0800777

5. Petron DJ, Greis PE, Aoki SK, Black S, Krete D, Sohagia KB, Burks R: Use of knee magnetic resonance imaging by primary care physicians in patients aged 40 years and older. Sports Health. 2010, 2:385-90. 10.1177/1941738110377420

6. Bannuru RR, Osani MC, Vaysbrot EE, et al.: OARSI guidelines for the non-surgical management of knee, hip, and polyarticular osteoarthritis. Osteoarthritis Cartilage. 2019, 27:1578-89. 10.1016/j.joca.2019.06.011

7. Cancienne JM, Werner BC, Luetkemeyer LM, Browne JA: Does timing of previous intra-articular steroid injection affect the post-operative rate of infection in total knee arthroplasty?. J Arthroplasty. 2015, 30:1879-82. 10.1016/i.arth.2015.05.027

8. Buchbinder R, Harris IA, Sprowson A: Management of degenerative meniscal tears and the role of surgery . Br J Sports Med. 2016, 50:1413-16. 10.1136/bmj.h2212

9. NHS Birmingham and Solihull CCG: policy for knee replacement surgery . (2018). Accessed: February 2, 2020: https://www.birminghamandsolihullccg.nhs.uk/about-us/publications/policies/105-policy-for-kneereplacement-surgery/file.

10. Robb C, McBryde C, Caddy S, Thomas A, Pynsent P: Oxford scores as a triage tool for lower limb arthroplasty lead to discrimination and health inequalities. Ann R Coll Surg Engl. 2013, 95:1-4. 